\title{
Complexity economics analysis of the international dry bulk freight rate system
}

\author{
Lingyu Song ${ }^{1, *}$ \\ ${ }^{1}$ Chengyi University College, Jimei University, 199 Jimei Avenue, Xiamen, China
}

\begin{abstract}
From the perspective of complexity economics, this paper studied the international dry bulk freight rate system, put forward the concept and structure model of the international dry bulk freight rate system and summarized its characteristics such as emergent properties, dynamicity, evolution and innovation, nonlinearity and non-equilibrium, which provides a new idea and research method for the study of the international dry bulk freight rate system.
\end{abstract}

\section{Introduction}

Most of the current literature study the dry bulk freight rate from the perspective of economics. Since the Baltic Dry Index (BDI) is the wind vane of the international dry bulk freight rate market, there are many studies on the fluctuation characteristics of BDI in the early stage. The recent studies mainly focus on the volatility spillover and guiding correlation of other economic variables on BDI, and explore the relationship between different submarkets within the international dry bulk market or among the international dry bulk market and other economic markets.

There are few studies on the research of international dry bulk freight rate market from the point of complex system. Simone cashili [1] and Li Zhenfu [2] proposed to study the world shipping system from the perspective of complex adaptive system. They believe that there are many adaptive and intelligent individuals such as ports and shipping enterprises. It is the interaction of these adaptive agents that makes the world shipping system share the characteristics of self-organization, emergent properties and non-linearity, indicating that the world shipping system is a typical complex system adaptive system. Zhang Houbao [3] proposed using Chaos Theory to analyze and verify the nonlinear properties of international dry bulk freight rate system and time series. The research results show that BDI time series has sensitive dependence on initial value, which means that the international dry bulk freight rate system is a nonlinear dynamic system with obvious chaotic characters. Chen Yancheng [4] and Tang Limin [5] put forward that the dry bulk shipping market is a complex time-varying system involving a variety of influencing factors, and the main factors causing freight rate fluctuation are the supply capacity of dry bulk shipping market, the trade volume of dry bulk cargo and shipping cost. After analyzing the interaction and influence of the above factors, they constructed the feedback relationship among these factors.

To sum up, there may be a more in-depth understanding of the international dry bulk freight rate market from a complex economic system in combination of the two perspectives. Based on this view, this paper will analyze the complex economic characteristics of the international dry bulk freight rate system from complexity economics, and establish a theoretical model of the feedback mechanism of the dry bulk freight rate system, which is of positive significance to analyzing and understanding the operation mechanism and evolution law of the international dry bulk freight rate market.

\section{The concept of dry bulk freight rate system and the complexity economics theory}

\subsection{Overview of complexity economics theory}

Brian Arthur proposes the term "Complexity Economics" covering a series of theories, hypotheses, tools, technologies and phenomena. He believes that economy is an evolutionary complex dynamic system in constant self-calculation, creation and renewal. It focuses on model formation, structural change, innovation, and the consequences of permanent creative destruction. Complexity economics is an economic theory beyond equilibrium [6-7].

In complexity economics, the economy shares the following models:

(1) Behaviour actors: make inductive decision with the limitation of incomplete information, error and deviation. They learn and adapt with time.

(2) Time: crucial and irreversible.

(3) Interaction: the networks of interaction among individuals change with time;

\footnotetext{
* Corresponding author: songlingyu@jmu.edu.cn
} 
(4) Evolution: the actions and strategies of actors are in continuous evolution;

(5) Innovation: innovation happens in the processes of variation, selection and amplification. Innovation is the main driving force of economy; it is discontinuous;

(6) Structure formation: structure is not given, but formed and recombined in the continuous evolution of a series of systems, norms and technological innovation;

(7) Auxiliary tools: computation

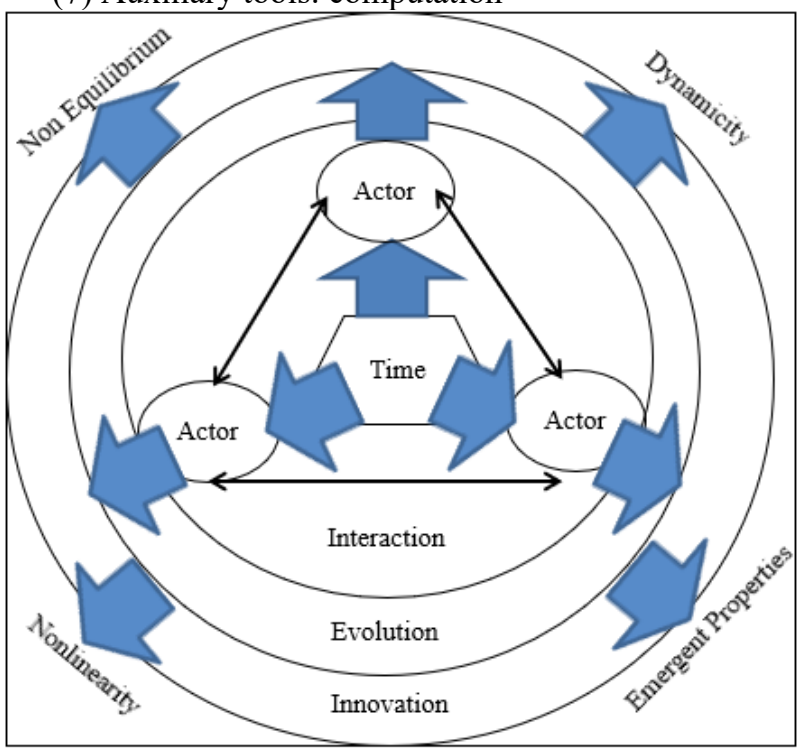

Fig. 1. Generated economic structure

Complexity economics holds the perspective that the "generated economic structure" presents the following characteristics (pls see Fig.1):

(1) Non equilibrium: disequilibrium is the natural state of economy;

(2) Dynamicity: economy is in a changing dynamic model;

(3) Nonlinearity: economy is a non-linear stochastic process with random events and natural positive feedback. The non-linear characteristics are expressed in the form of positive feedback, which comes from the increasing returns, and its results are unpredictable and often locked. Depending on the historical path taken, it's not necessarily the most effective in economy. So the economic system is full of historical contingency;

(4) Emergent properties: the emergence of "generated order" emphasizes the importance of meso economy, while the macro level is the result of action and interaction between actors at the micro level. Evolution and innovation lead to changes in order, economic structure and system complexity.

The purpose of conducting complexity economics research on dry bulk freight system is to deeply understand the mechanism creating these economic models.

\subsection{Definition of dry bulk freight rate system}

At present, though in the previous literature, the concept of dry bulk freight rate system is mentioned from time to time, no scholars have made a clear definition to it. From the perspective of complexity economics, this paper holds that the international dry bulk freight rate system refers to the two main actors that directly affect the formation of international dry bulk freight rate in the world, that are dry bulk shipping enterprises and dry bulk cargo owner enterprises. The technology carriers that the actors use are ships and ports, other external nodes providing services for them such as port enterprises, shipyards, brokers and shipping agency, the unified whole composed of economy, politic, society and culture, and the environment like government regulation, political events, climate change and ecological protection[8].

In this whole, the actors of the international dry bulk freight rate system, as well as the actors with external nodes and environment make interaction, influence and adaption among each other and adjust their own behavior in a complex way, resulting in different feedback structures and many subsystems at the meso level, such as shipping demand subsystem, capacity supply subsystem, new ship building subsystem.

\subsection{Internal mode and behaviour actors of dry bulk freight rate system}

As the main actors of dry bulk freight rate system, shipping companies and traders will seek and summarize the rules from the past experience and evidence to make inductive decision. For example, when a shipping company makes a new shipbuilding investment decision, they will consider current freight rate, capacity, new ship volume, ship dismantling volume, shipping demand (trade volume), new ship price, second-hand ship price and the ratio, shipbuilding cost, oil price, market cycle, investor expectation and risk preference, etc., from which they will consider whether to build a new shipbuilding. Besides, the relative importance of rules of thumb will change with time. However, they are limited by incomplete information, errors and deviations. For instance, when shipping companies decide to invest in new shipbuilding, they cannot immediately know the new shipbuilding orders of other enterprises, making them overestimate the impact of the surge in shipping service demand, and partially ignore the endogenous investment response of other shipping companies. Once they realized the low returns followed, the shipping companies had over invested in the boom period and paid too high prices for new ships, leading to the following long-term recession caused by too many orders. However, they will learn from it and adapt to it. More ship-owners will choose to build new ships in the recession period, leading to the extension of the recession period. The experience or rules that shipowners considered are roughly the same, which makes them have regular behavior. But at the same time, with different beliefs, they continue to learn and adapt, and adjust their decisions.

As the main actors, the interaction between traders and shipping companies has formed the dry bulk freight rate, but in the meanwhile, the change of freight rate also forces them to constantly adjust their business and technical strategy as shown in Fig.2. At the same time, the dry bulk freight rate system needs ships and ports as 
the carrier of marine transportation of goods, and they are also responsible for the technological innovation of dry bulk freight rate system. In turn, the technological innovation continuously pushes back the improvement of the business model. Some main actors are eliminated through the evolutionary selection mechanism, and new actors are emerging at the same time.

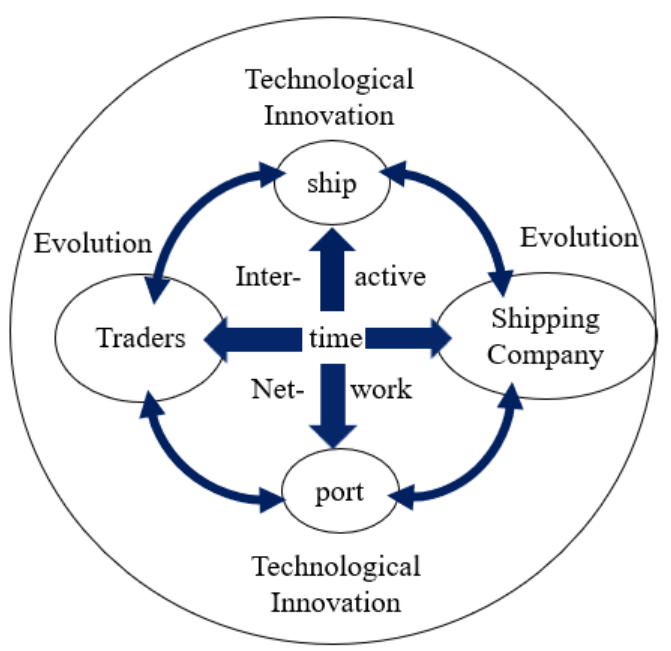

Fig. 2. Internal Mode of dry bulk freight rate system

\subsection{Feedback structure of dry bulk freight rate system}

\subsubsection{Shipping demand subsystem - a positive feedback with short delay}

The fluctuation of economy and trade will directly drive the change of related dry bulk shipping demand. Facing a rapid economic development, traders will exaggerate the demand for goods, or they will delay the demand for goods transportation under adverse economic growth. The exaggerated demand for goods transportation leads to the rise of freight rate, and the rise (or fall) of freight rate usually leads to the advance purchase (or selective purchase) of transportation services by traders [9]. Taking the rise of freight rate as an example, more and more traders transport the future trade in advance when the freight rate is increasing. The increase of sea freight volume leads to an increasing of transport price, further aggravating traders' market psychological expectations of rising price. Herd Effect pushes the traders to expand the sea freight volume. The positive feedback promotes the exponential rise of freight rate. And in turn, when the freight rate falls, trader will conduct purchase selectively. The dry bulk traders will delay the time of shipping orders and reduce the freight volume, which further causes the continuous decline of freight rate.

There will be a certain time delay for traders' response to the rise or fall of freight rate. Based on their own needs, they will adjust the timing and volume of shipping orders according to the changes of market freight rate. Limited by trade contracts, the time delay caused by adjustments is short.

\subsubsection{Current shipping capacity supply subsystem - a negative feedback with limited time adjustment}

The demand for dry bulk shipping can increase or decrease sharply due to the traders' early purchase or selective purchase. However, the utilization and turnover efficiency of existing transport capacity have the scope limit of up and down adjustment, and there is also a certain adjustment time delay in the capacity turnover and utilization efficiency.

Taking the rise of freight rate as an example, the dry bulk ship-owners adjust the turnover efficiency of existing ships, increase the sailing speed of ships and the loading efficiency, improve the utilization efficiency of existing transport capacity. The dismantling of ships is almost stopped, and all old ships are put into operation, increasing the existing transport capacity. The negative feedback mechanism can ease the rise of freight rate. However, if the demand increase sharply, the supply of transport capacity fails to surge with the demand with the utilization and turnover rate of existing transport capacity reaching the limit. Under this situation, the negative feedback mechanism fails to work, instead, the positive feedback mechanism of dry bulk transport demand dominates and the freight rate continue to rise.

If the freight rate falls, ship-owners can reduce the supply of transport capacity by dismantling old ships and reducing ship speed. The reduction of transport capacity supply can ease the drop of freight rate. However, if the demand continues to fall, with limited adjustment capacity, the supply of transport capacity exceeds the demand, and the positive feedback mechanism of transport demand occupies the dominant position, which makes the freight rate continue to decline.

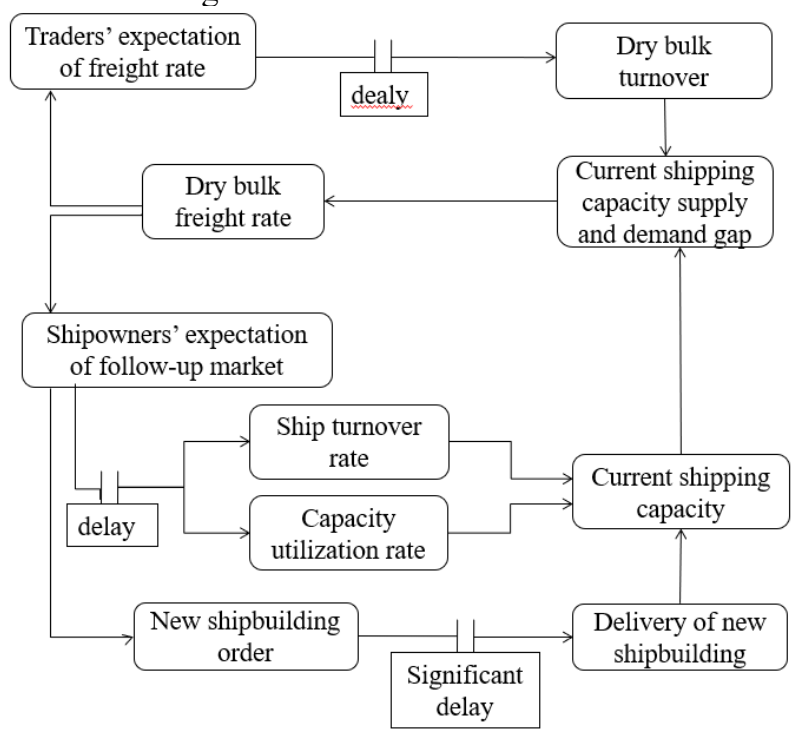

Fig. 3. Feedback structure of dry bulk freight rate system 


\subsubsection{Shipping capacity supply subsystem of new shipbuilding - a negative feedback with significant time delay}

With the shortage of transport services in the market, traders, on the demand side, usually exaggerate their demand to fight for transport prices and transport opportunities, making shipping enterprises receive untrue and excessive demand information. Ship-owners start to order new ships to improve their transport capacity according to the trend of demand change, but the time from order construction to operation is delayed significantly, which means the response of new shipbuilding capacity supply is in an obvious lag.

The new transport capacity cannot immediately put into use in transport market in the short term, so that the negative feedback cannot work. However, the trade demand is constantly pushed up owing to the cargo owners' exaggerated demand and opportunistic behavior. As a result, the demand for dry bulk cargo transportation is far greater than the supply, and the freight rate continues to rise, making the ship-owners more and more optimistic about the follow-up market. The price rise will stimulate the emotion, leading to a large number of new shipbuilding investment. In the short term, shipbuilding orders are filled to capacity, causing a backlog of orders because of limited production capacity. When the adjustment time of transportation capacity is exceeded over time, the new shipbuilding will be put into the market in large quantities. An increase of capacity reduces the freight rate. If the demand continues to go up, the increase of freight rate will be slowed down; if the demand is reduced, the demand will fall. Coupled with the increase of the capacity, the freight rate will drop sharply.

The above three kinds of meso level subsystem feedback results generated by the interaction of micro level actors occupy a dominant position with the change of their relative strength over time, which leads to the different macro structures in constantly generating and restructuring of dry bulk freight rate system.

\section{Complexity economic analysis of dry bulk freight rate system}

\subsection{Emergent properties of dry bulk freight rate system}

The interaction of the actors in the international dry bulk freight rate system on the micro level forms the structure of the subsystems at the meso level. The different subsystems structure takes different positions with time, which leads to the emergence of the macro level of the system, that are the partial distribution of freight index, price oscillation and periodic cycle.

The skew distribution refers to the abnormal distribution of the BDI time series of the dry bulk freight index in high apex and thickness tail, which means that there is an extreme value that greatly deviates from the mean value in the BDI time series, indicating a sharp rise and fall in the international dry bulk freight rate market.
The reason for a violent fluctuation of BDI is that there is a positive feedback subsystem in BDI system, which promotes the BDI to go up or down in a single direction. However, there is more than one negative feedback subsystem with time delay, restraining BDI from an unlimited rise or fall, so that it would represent a homing behavior.

The interaction between different subsystems makes the international dry bulk freight rate system have a periodic cycle. There are three obvious periodic clusters: 3-5 years of Kichen Cycle, 7-10 years of Zugra Cycle and 16-20 years of Kuznets Cycle. The reason is that there are feedback subsystems with different adjustment speed in the dry bulk freight rate system. The difference of adjustment speed of each subsystem, or we said the length of time delay should be the reason for the periodic cycle of the dry bulk freight rate system.

For the supply and demand subsystem of dry bulk freight rate system, the supply subsystem has strong rigidity in a short time that the transport capacity will not change greatly. However, the demand subsystem is always changing. Though the time delay of adjusting demand fluctuation is the shortest, when the supply of transport capacity doesn't change in time, the fluctuation of shipping demand will play a leading role, and then cause a cyclical fluctuation of freight rate. In the medium term, when the new shipbuilding is put into use, or it is requisitioned or dismantled, the fluctuation of transportation capacity caused by the delay of new shipbuilding lead to the periodic fluctuation of freight rate. In the long run, the fluctuation of global economic situation besides the international dry bulk freight rate system, that is, exogenous input, determines the periodic fluctuation of freight rate. At present, the innovation of new shipbuilding technology shortens the shipbuilding time, as well as the medium cycle. The short period and medium cycle also overlap. However, the long-term cycle is not obvious due to the slowdown of global economic growth, so the three periods of dry bulk freight transportation rate system cannot be divided clearly.

To sum up, there are three fundamental reasons for the emergence: firstly, the behavior regularity of the participants in the system; secondly, the feedback structure of each subsystem at the meso level. Different feedback structures occupy a dominant position at different times under different circumstances; finally, the external input of the system, that is, the change of the external global economic and trade environment.

\subsection{Dynamicity of dry bulk freight rate system}

Dynamic means that the freight rate system will always change with time. The most obvious feature of the dynamic system is the system structure that the stock and flow interconnected in a complex feedback loop. After the structure emerged and lasted for a certain period of time, due to the exploration and attempt of each actor, the original structure disintegrated and a new economic structure was established. Its continuous dynamic evolution came from different system structures under 
different situations in a given time when the feedback loop with the largest restriction or constraint dominates.

Another important part of dynamic system is time delay. In the short term, the negative feedback of new shipbuilding capacity supply plays a small role, while the negative feedback of current capacity supply and the positive feedback of demand work at the same time, among which, the positive feedback of demand leads the dominant position like the rapid rise of BPI index just after the Spring Festival. In the medium term, the supply negative feedback and demand positive feedback of new shipbuilding capacity work at same time. If the market is in a low oscillation with insufficient demand, the supply negative feedback of new shipbuilding capacity will occupy a dominant position.

\subsection{Evolution and innovation of dry bulk freight rate system}

This is the coevolution of the whole dry bulk freight rate system, which exists in each stage of the dry bulk freight rate system evolving with time.

Affected by the changes of the world political, economic development and scientific and technological progress, the dry bulk freight rate system will continue to evolve. At the same time, the actors in the system are constantly adjusting the way of exchange with other actors and the external environment to adapt to the changing environment. This is the coevolution of the whole dry bulk freight system. This adaptive coevolution process exists in each stage of the dry bulk freight system evolution with time.

The globalization in the past few decades lead to a surge in trade volume, especially the bulk trade of raw materials, requiring larger demand for the dry bulk cargo ship. The operation action and strategy of using the ship as the carrier of transportation are evolving. When a certain actor, such as a ship company, adopts the strategy of building large ships, the efficiency of ship enlargement increases, benefiting the shipping companies that adopt this strategy, and then, a coevolutionary process of variation, selection and amplification happens in the dry bulk freight rate system. For example, the scale effect of ship large-scale is reflected in the BPI sub ship type index of Panamanian ship. The standard ship type is changed from 74000 DWT to 82000 DWT.

The ship maximization requires faster loading and unloading speed and higher efficiency, while the requirements of traders and shipping companies for high speed and efficiency force port enterprises to constantly update their own facilities, speed up loading and unloading speed, and strive for a more important port position on the route, which brings about the innovation of automatic loading and unloading technology and the improvement of port management efficiency. Its functions are also constantly transforming and upgrading, and the port is changing from generation to generation, which is the performance of port development and evolution.
As another example, the shortening of shipbuilding cycle is led by shipping companies, forcing shipyards to innovate shipbuilding technology, reducing the time required for new shipbuilding from two or three years to eight months now. At present, the evolution directions such as automated ships, $5 \mathrm{~g}$ applications, block chain technology all undergone natural selection, and then form their evolution direction.

In addition to the evolution which brought about in the pursuit of efficiency within the system, changes in the external environment will also promote the actors to adjust themselves, such as environmental pressure. The $0.5 \%$ Sulfur Limit on marine fuel of the International Maritime Organization comes into effect, improving the clean standard of marine fuel. Therefore, some dry bulk transportation enterprises install desulfurization towers for ships or order new energy ships under high oil prices, which promotes development of the marine technology.

In addition to technological innovation, actors also continuous evolution in terms of strategy. Different types of actors compete and cooperate with each other, such as the cooperation between shipping companies and port enterprises, and long-term transportation agreements signed between traders and shipping companies. Innovation is the main driving force of economy, while evolution and innovation lead to changes in order, economic structure and complexity of dry bulk freight rate system.

\subsection{Nonlinearity of dry bulk freight rate system}

The nonlinear of dry bulk freight rate system is reflected in the occurrence of random events, namely, the sensitivity dependence on initial value. It means that the long-term future behavior of the international dry bulk freight rate system is extreme sensitive to the minor changes or deviations of initial conditions. The random occurrence of events determines the initial value, so the dry bulk freight rate system is full of accidents.

Moreover, the non-linearity of the dry bulk freight system is also expressed with the form of positive feedback, which comes from the increasing returns and reflects in the positive feedback of the shipping demand subsystem. When there happens a random event that promotes shipping demand, with time going by, the increasing returns will amplify the cumulative effect of this event on the random selection results.

Finally, the nonlinearity of the dry bulk freight rate system is also reflected in the long memory characters, which means that the past freight rate of the system has a strong continuous impact on its future freight rate changes. The future freight rate is affected not only by the current freight rate, but also by the past freight rate. In the dry bulk freight rate system, after receiving the new market information, the actors, including dry bulk ship-owners, traders, etc. will not immediately make changes to deal with the new market information, but will refer to the practice of other actors, which means that the actors will react with lag accumulation to the previously ignored information, so that the response of dry bulk freight rate presents a dynamic "pile up", that is, 
it presents "persistence or long memory" when it fluctuates[9]. If the freight index has an upward (downward) trend in the previous stage, it will continue to maintain an upward (downward) trend in the next stage.

\subsection{Non equilibrium of dry bulk freight rate system}

The non-equilibrium state is the normal state of the dry bulk freight rate system. Actors such as shipping companies and traders can constantly adjust their market behaviors, transportation decisions, pricing strategies and future forecasts by considering the possible results of their own behaviors, so as to adapt to the overall model composed of these market behaviors, trading decisions, pricing strategies and future forecasts. When the actor reacts, the general pattern changes, and vice versa, the actor has to change his behavior and strategy according to the general pattern. This kind of reaction further changes the result, which needs them to adjust again. It is the so-called recursive cycle, which makes the dry bulk freight system have endogenous disequilibrium. There are two main reasons for the emergence of endogenous disequilibrium: first, the uncertainty of decision-making, and second is the technological innovation or technological change.

The non-equilibrium phenomena in the dry bulk freight rate system are in the following kinds: first, the self-strengthening of transportation price changes or the spontaneous formation of "bubbles and collapses"; the second is cluster fluctuation referring to the phenomenon of random alternations between low and high fluctuation periods, which is called "Generalized AutoRegressive Conditional Heteroskedasticity", or GARCH behavior.

In the complex dynamic system, besides the transformation of the dominant position of feedback structure, there occurs inflection point, which means that there must be a major interference event or one cumulative effect reaching the critical value. The policy environment and industry cycle in macro level may cause a big inflection point; on the micro level, if the development speed of enterprises or the confidence of investors changes, inflection point may also occur. For example, the sharp decline of BDI index in 2008 was due to the economic crisis in the external environment, making a slowdown of trade and the sharp reduction of transportation demand. However, the huge orders of new shipbuilding and the gradual delivery of new ships leads to a drop in freight rates in oversupply, further aggravating the selective cargo transportation behavior of ship-owners, and making ship-owners receive bad market information. Ship-owners' investment confidence changed, and psychological impact makes panic accumulate. In addition, information filtering, social pressure and financial pressure also greatly increased the accumulation of panic, resulting in a dramatic fall in freight rates.

\section{Conclusion}

Based on evolution theory and complex adaptive system theory, the complexity economics requires us to detach from the curve of the rise and fall of dry bulk freight rate, and consider the international dry bulk freight market as a whole, or a system. It provides a new perspective for the study of international dry bulk freight rate system as a whole, and it will serve as a powerful tool for the indepth study of international dry bulk freight rate system.

The conclusions are as follows:

(1) The international dry bulk freight rate system is a unity composed of the actors and environment related. From the overall perspective of complexity economics, it is of positive significance to analyze and understand the development and evolution of the dry bulk freight rate system.

(2) This paper established the concept map model of the internal mode of international dry bulk freight rate system, laying a foundation for the in-depth research of freight fluctuation of dry bulk freight rate system.

(3) The international dry bulk freight rate system features in emergent properties, dynamicity, evolution and innovation, nonlinearity and non-equilibrium, showing that international dry bulk freight rate system is a typical evolution system of complexity economics.

\section{References}

1. S. Caschili, F. Romana Medda, Inter-disciplinary Description of Complex Systems, 10(1):1-15 (2012)

2. Li Zhenfu, Jiang Shufei, Xu Mengqiao, Shi yanlei, Zhang Xiaoling, Logistics technology, 34 (01): 8-10 (2015)

3. Zhang Houbao, Ding Yi, Lin Guolong, Henan science, 31 (10): 1675-1679(2013)

4. Chen Yancheng, Lin Guolong, Hu Zhihua, Journal of Wuhan University of Technology (traffic science and Engineering Edition), 36 (04): 799-803(2016)

5. Tang Limin, Zhang Mengyao, Wang pan, Journal of Dalian Maritime University, 44 (02): 95-103(2018)

6. Brian Arthur. Complex Economics: a new framework of economic thought, Zhejiang People's publishing house (2018)

7. Eric beinhock, The origin of wealth, Zhejiang People's publishing house (2019)

8. Jiang Shufei, Dalian Maritime University (2015)

9. Wan Jiuwen, Lu Jing, Wei Fang, Gong Xiao, Journal of Dalian Maritime University, 36 (03): 31$34+41(2010)$ 